\title{
Validation of an LC-MS/MS method to determine five immunosuppressants with deuterated internal standards including MPA
}

\author{
Armin Buchwald*, Karl Winkler and Thomas Epting
}

\begin{abstract}
Background: Therapeutic drug monitoring of immunosuppressive drugs in organ-transplanted patients is crucial to prevent intoxication or transplant rejection due to inadequate dosage. The commonly used immunoassays have been gradually undergoing replacement by mass spectrometry, since this physical method offers both a higher sensitivity and specificity. However, a switch should be carefully considered because it is a challenging procedure and needs to be thoroughly validated.

From an economic perspective it is reasonable to include mycophenolic acid into the assay, because this saves the necessity for an additional measurement. However, to date very few validation protocols for the measurement of immunosuppressants, including mycophenolic acid, are available. In order to adequately compensate for matrix effects, the use of stable isotope labeled internal standards is advisable. Here, the authors describe a single method suitable for the quantification of cyclosporine A, tacrolimus, sirolimus, everolimus and mycophenolic acid, based on deuterated internal standards.

Methods: Plasma proteins were precipitated with zinc-sulfate, followed by an online solid phase extraction in the flow-through direction. Chromatographic separation was performed by a c18-phenyl-hexyl column. For subsequent mass spectrometric analysis stable-isotope-labeled internal standards were used. Results were available after 3.5 minutes.

Results: Low quantification limits (accuracy: $104-118 \%$ ) and linearity resulted in $2-1250 \mathrm{ng} / \mathrm{ml}$ for cyclosporine A; $0.5-42.2 \mathrm{ng} / \mathrm{ml}$ for tacrolimus; $0.6-49.2 \mathrm{ng} / \mathrm{ml}$ for sirolimus; $0.5-40.8 \mathrm{ng} / \mathrm{ml}$ for everolimus and $0.01-7.5 \mu \mathrm{g} / \mathrm{ml}$ for mycophenolic acid. Intra-assay precision revealed a coefficient of variation (CV) of $0.9-14.7 \%$, with an accuracy of $89-138 \%$. The CV of inter-assay precision was $2.5-12.5 \%$, with an accuracy of $90-113 \%$. Recovery ranged from 76.6 to $84 \%$. Matrix effects were well compensated by deuterated internal standards.

Conclusions: The authors present a fast, economical and robust method for routine therapeutic drug monitoring comprising five immunosuppressants including mycophenolic acid.
\end{abstract}

\section{Background}

Therapeutic drug monitoring (TDM) of immunosuppressive drugs in organ-transplanted patients is vitally important to prevent intoxication or rejection due to incorrect dosage. New therapeutic regimens combine immunosuppressants with different intracellular targets to lower blood concentrations and prevent undesired side effects [1-3]. This practice requires a precise and

\footnotetext{
* Correspondence: armin.buchwald@uniklinik-freiburg.de Division of Clinical Chemistry, Department of Medicine, University Medical Center Freiburg, Hugstetterstrasse 55, 79106 Freiburg, Germany
}

accurate analytical method, especially for the lower ranges of concentrations. With regards to sensitivity immunoassays often fail to meet clinical needs, due to their restricted detection limits. Moreover, they are vulnerable to cross-reactions against pharmacologically inactive metabolites, resulting in limited specificity and possibly false results [4].

In order to minimize imprecision at low drug concentrations, elaborate sample preparation is required to separate the molecules of interest from the patient's blood matrix molecules [5]. Any remaining matrix can adversely affect the efficiency of ionization and lead to
C Biomed Central

(c) 2012 Buchwald et al; licensee BioMed Central Ltd. This is an Open Access article distributed under the terms of the Creative Commons Attribution License (http://creativecommons.org/licenses/by/2.0), which permits unrestricted use, distribution, and reproduction in any medium, provided the original work is properly cited. 
erroneous results. Thus, matrix effects need to be identified and compensated by internal standards (IS). Since stable isotope labeled, structurally analogous isoforms are the most appropriate controls for matrix compensation [6,7], deuterated equivalents are about to replace the common IS ascomycin, cyclosporine D (CSD) [8-10] and carboxy butoxy ether of mycophenolic acid (MPAC).

Cyclosporine A (CSA), tacrolimus (TAC), everolimus (EVE) and sirolimus (SIR) are measured in whole blood, whereas mycophenolic acid (MPA) is determined in plasma $[11,12]$. Several methods have been reported to measure these drugs using different techniques for sample preparation and high pressure liquid chromatographic (HPLC) schedules [8,13-15]. However, these applications lack either appropriate IS or MPA to complete the analytical spectrum. Moreover, the separation of the main MPA metabolite mycophenolic acid glucuronide (MPAG) is essential for mass spectrometric analysis because MPAG can undergo in-source fragmentation to MPA via loss of the glucuronic acid moiety [11], which in the case of coelution is determined as MPA.

Generally, sample preparation consists of precipitation with a mixture of zinc-sulfate, organic solvent (methanol, acetonitrile or acetone) and IS, usually CSD, ascomycin and MPAC. The method proposed by Koster et al. for example precipitates proteins using $\mathrm{zn}$-sulfate only for CSA and TAC, but not for SIR and EVE [16]. This approach has the disadvantage of requiring separate runs to get all of the analytes quantified.

Adding water before precipitation prevents sample clotting and improves extraction efficiency [17]. However, due to higher dilution this procedure needs a highly sensitive mass spectrometer.

One step that most sample preparation protocols described in the literature have in common is that after precipitation debris and contaminants must be removed, before the extracts undergo testing.

This necessitates two-dimensional chromatography with a 6 or 10 port switching valve. First, the sample is injected into the extraction column with aqueous extraction buffer containing a low concentration of organic solvent, after which it is then flushed at high flow rates (up to $5 \mathrm{ml} / \mathrm{min}$ ). After valve switching, the organic buffer usually is run in back-flush mode to elute the analytes to the analytical column.

Another frequently used method is solid phase extraction in offline mode, which resembles the online procedure. The main difference is the desiccation of the eluted analytes under an airstream, followed by reconstitution in analytical buffer, before the sample can be injected into the HPLC-device.

This paper presents the complete validation of a single LC-MS/MS method for five immunosuppressants, including MPA, based on protocols described by Annesley et al. [17] and Seger et al. [15]. CYA, TAC, SIR and EVE were analyzed simultaneously in a single analytical run, whereas MPA was analyzed separately due to its preparation from plasma. To our knowledge this is the first application using corresponding deuterated IS (CSA-d4, TAC- ${ }^{13} \mathrm{C}-\mathrm{d} 2$, EVE-d4, SIR- $-{ }^{13} \mathrm{C}-\mathrm{d} 3$, and MPAd4) for each immunosuppressant and identical HPLC running conditions for all analytes. The use of an online solid-phase extraction in straight flush mode makes this application suitable for the determination of MPA as well.

\section{Methods}

The protocol described in this paper follows internationally accepted guidelines (NCCLS, FDA) for the validation of in-house methods $[18,19]$.

Patient material was used in anonymous form in consent with the ethics committee of the University Medical Faculty, Freiburg. According to the guidelines of ICMJE and WHO a study registration was not required.

\section{Reagents and materials}

Immunosuppressants CSA, TAC, SIR and MPA, as well as water, methanol, acetic acid and ammonium acetate in LC-MS quality were purchased from Sigma-Aldrich (Munich, Germany). EVE was kindly provided by Novartis (Basel, Switzerland). MPAC was a kind gift of Roche Palo Alto, California. Zinc sulfate heptahydrate was supplied by Merck (Darmstadt, Germany).

Deuterated standards CSA- $\mathrm{d}_{4}, \mathrm{TAC}-{ }^{13} \mathrm{C}-\mathrm{d}_{2}, \mathrm{EVE}-\mathrm{d}_{4}$ and MPA- $\mathrm{d}_{3}$ were obtained from Toronto Research Chemicals (Ontario, Canada) and $\mathrm{SIR}_{-}{ }^{13} \mathrm{C}-\mathrm{d}_{3}$ from Alsachim (France). For calibration and quality control the 6Plus1 multilevel calibration kit and 4-level whole-blood controls of Chromsystems (Munich, Germany) were used. Calibrator and controls for MPA were provided by Recipe (Munich, Germany).

\section{Sample preparation}

The concentrations of IS were adjusted to the area under the curve (AUC) of calibrator 2 (MPA: control 2) and were $50 \mu \mathrm{g} / \mathrm{ml}$ for CSA- $\mathrm{d}_{4}, 0.8 \mu \mathrm{g} / \mathrm{ml}$ for TAC- ${ }^{13} \mathrm{C}$ $\mathrm{d}_{2}, 0.8 \mu \mathrm{g} / \mathrm{ml}$ for SIR- ${ }^{13}{ }^{13}-\mathrm{d}_{3}, 0.25 \mu \mathrm{g} / \mathrm{ml}$ for EVE- $\mathrm{d}_{4}$ and $6 \mu \mathrm{g} / \mathrm{ml}$ for MPA- $\mathrm{d}_{3}$.

The working solution was prepared fresh daily and consisted of $30 \mathrm{ml}$ methanol, $15 \mathrm{ml}$ zinc sulfate solution $(0.1 \mathrm{M})$ and $100 \mu \mathrm{l}$ IS-Mix - sufficient for the treatment of 120 samples.

Samples were prepared according to the protocol described by Annesley et al. [17] and Seger et al. [15] with modifications as follows: $50 \mu$ EDTA whole blood were taken for each sample to determine CSA, TAC, SIR and EVE, but $50 \mu \mathrm{l}$ EDTA plasma was used to 
analyze MPA. In the instances of patients treated with a combination therapy including MPA, an aliquot of the EDTA sample was taken for plasma extraction just before the preparation of whole blood for CYA, TAC, SIR or EVE analysis. The latter were analyzed simultaneously in a single analytical run. Therefore, the plasma and whole blood preparations had to be injected separately. However, apart from different starting material, all preparation steps and analytical conditions were identical.

Samples, controls and calibrators were first mixed on a roller, hemolysed by adding $125 \mu \mathrm{l}$ of water, thoroughly vortexed and then incubated for two minutes at room temperature (RT). The next step was an ultrasonic bath for $20 \mathrm{sec}$ (omitted in the case of MPA). Initially, time dependent progression of hemolysis was confirmed by microscopy: immediately after the addition of water; then after 2 minutes of incubation with water and finally after 2 minutes incubation with water plus $20 \mathrm{sec}$ of ultrasonic treatment. After the addition of $375 \mu \mathrm{l}$ working solution samples were vortexed, kept for 10 minutes at RT and centrifuged at $15000 \mathrm{~g}(10 \mathrm{~min})$. The supernatants were subsequently transferred to autosampler vials. The described procedure corresponds to a final dilution of 1:11 (v/v). To clarify whether matrix effects can be affected by a different dilution ratio, dilutions of 1:3 and 1:21 were carried out in parallel.

\section{Liquid chromatography}

For chromatographic separation, a Shimadzu Prominence HPLC system equipped with three isocratic pumps was used. The injection volume was $2 \mu \mathrm{l}$ for MPA and $20 \mu \mathrm{l}$ for the remaining immunosuppressive drugs. For online purification a solid phase extraction column (SPE) Poros R1/20, $2.1 \mathrm{mmD} \times 30 \mathrm{~mm}, 20 \mu \mathrm{m}$ (Applied Biosystems, Darmstadt, Germany) was chosen. A phenyl-hexyl reversed phase C18 column Zorbax Eclipse XDB (Agilent, Waghäusel, Germany) $3.0 \times 75$ $\mathrm{mm}, 3.5 \mu \mathrm{m}$ at $60^{\circ} \mathrm{C}$ was used as the analytical column.
Column switching was controlled by a multi-channel valve (Valco 10 port 2 position valve) according to the pattern shown in Figure 1.

After injection, the SPE column was loaded for one minute with aqueous eluent $(90: 10 \mathrm{v} / \mathrm{v}$, water/methanol containing $10 \mathrm{mM}$ ammonium acetate and $0.1 \%$ acetic acid) at a flow rate of $5.0 \mathrm{ml} / \mathrm{min}$. After valve switching organic molecules were eluted to the analytical column with methanolic buffer $(97: 3 \mathrm{v} / \mathrm{v}$, methanol/water, 10 $\mathrm{mM}$ ammonium acetate and $0.1 \%$ acetic acid) at a flow rate of $0.9 \mathrm{ml} / \mathrm{min}$. During that time both columns were connected in series, so that the flow direction corresponded to the loading direction. After switching the valve to the starting position, the Poros column was rinsed with pure methanol at $1 \mathrm{ml} / \mathrm{min}$, to remove any remaining organic molecules and prevent carry-over contamination.

A $40 \mathrm{sec}$ conditioning phase of the SPE column with aqueous buffer at a flow rate of $5 \mathrm{ml} / \mathrm{min}$ completed the run to a total analysis time of $3.5 \mathrm{~min}$.

\section{Mass spectrometry}

Mass spectrometric analysis was run on an API 4000 (AB Sciex, Darmstadt, Germany) equipped with an electrospray ionization interface (ESI). Analyte-specific voltage settings and ion-source adjustment were set manually, the former after automatic optimization. Gas settings in $\mathrm{ml} / \mathrm{min}$ were: Collision gas: 7 , curtain gas: 20 , ion source gas 1: 50 , ion source gas $2: 75$, ion spray voltage: $5500 \mathrm{~V}$, and temperature: $400^{\circ} \mathrm{C}$. Ammonium adducts of each analyte were detected in positive ion mode by multiple-reaction-monitoring. Mass transitions can be found in Table 1. There is no qualifier for MPAG since this metabolite was not quantified but only monitored.

\section{Validation methods}

In order to determine the limit of quantification (LOQ) blank calibrator was spiked with low calibrator to obtain

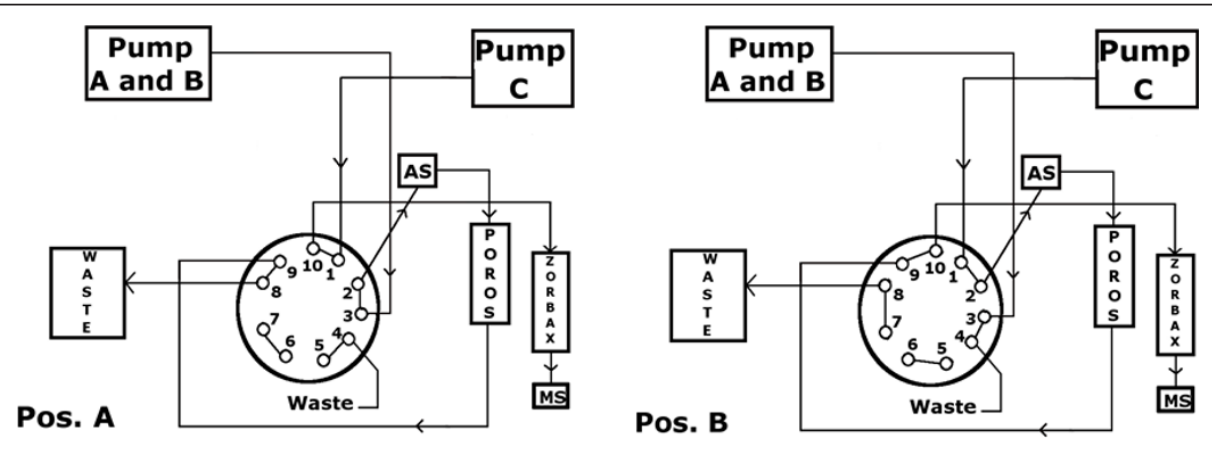

Figure 1 Column Switching. Pos A: Conditioning of the analytical column and loading of SPE column with aqueous buffer containing 10\% methanol. Pos B: Elution from the analytical column ( $97 \%$ methanolic solvent) and rinsing step of precolumn with pure methanol. 
Table 1 Mass transitions of analytes and internal standards

\begin{tabular}{|c|c|c|c|c|c|}
\hline $\begin{array}{l}\text { Internal Standard } \\
\text { Quantifier }\end{array}$ & Q1 & Q3 & $\begin{array}{c}\text { Analyte } \\
\text { Quantifier }\end{array}$ & Q1 & Q3 \\
\hline Cyclosporine A-d 4 & 1224.0 & 1206.9 & Cyclosporine A & 1219.9 & 1203.0 \\
\hline Tacrolimus ${ }^{13} \mathrm{C}-\mathrm{d}_{2}$ & 824.6 & 771.6 & Tacrolimus & 821.5 & 768.4 \\
\hline Sirolimus- ${ }^{13} C-d_{3}$ & 935.6 & 864.6 & Sirolimus & 931.6 & 864.7 \\
\hline Everolimus- $\mathrm{d}_{4}$ & 979.6 & 912.6 & Everolimus & 975.6 & 908.5 \\
\hline Mycophenolic acid- $d_{3}$ & 341.2 & 210.1 & Mycophenolic acid & 338.2 & 207.1 \\
\hline Mycophenolic acid glucuronide & 513.6 & 207.2 & & & \\
\hline Carboxy butoxy ether of mycophenolic acid & 438.2 & 303.0 & & & \\
\hline $\begin{array}{c}\text { Internal Standard } \\
\text { Qualifier }\end{array}$ & Q1 & Q3 & $\begin{array}{l}\text { Analyte } \\
\text { Qualifier }\end{array}$ & Q1 & Q3 \\
\hline Cyclosporine $\mathrm{A}-\mathrm{d}_{4}$ & 1224.0 & 1188.8 & Cyclosporine A & 1219.9 & 1185.0 \\
\hline Tacrolimus ${ }^{13} \mathrm{C}-\mathrm{d}_{2}$ & 824.6 & 789.4 & Tacrolimus & 821.5 & 786.5 \\
\hline Sirolimus ${ }^{13} \mathrm{C}-\mathrm{d}_{3}$ & 935.6 & 882.4 & Sirolimus & 931.6 & 882.4 \\
\hline Everolimus- $\mathrm{d}_{4}$ & 979.6 & 930.5 & Everolimus & 975.6 & 926.6 \\
\hline Mycophenolic acid- $d_{3}$ & 341.2 & 306.3 & Mycophenolic acid & 338.2 & 210.1 \\
\hline Carboxy butoxy ether of mycophenolic acid & 438.2 & 195.0 & & & \\
\hline
\end{tabular}

the desired concentration. All calibrators and controls were reconstituted according to the manufacturer's instructions, aliquoted and then frozen at $-20^{\circ} \mathrm{C}$. The LOQ was defined as the lowest measurable concentration which could be determined with a CV below $10 \%$. For this, drug free blood was spiked with calibrator 1 (lowest concentration) to a concentration of $2 \mathrm{ng} / \mathrm{ml}$ for CSA, $0.5 \mathrm{ng} / \mathrm{ml}$ for TAC, $0.65 \mathrm{ng} / \mathrm{ml}$ for SIR and 0.55 $\mathrm{ng} / \mathrm{ml}$ for EVE. The final concentrations varied due to a calibrator-saving common preparation step.

For MPA, plasma calibrator was prepared with drugfree plasma to a final concentration of $0.01 \mu \mathrm{g} / \mathrm{ml}$. Aliquots of all three stock solutions were frozen.

Samples for the detection of linearity were prepared in drug-free blood pooled with calibrator 6 (highest concentration) at three different ratios (1:2, 1:4 and 4:1), to obtain a graph consisting of five data points. Native calibrator level 6 and blank blood served as starting points. In the case of MPA, drug-free plasma was spiked with mycophenolate to a concentration of $7.5 \mu \mathrm{g} / \mathrm{ml}$ and prepared in the same way. Final concentrations were as follows: CSA (blank; 224; 448; 672 and $896 \mathrm{ng} / \mathrm{ml}$ ); TAC (blank; 10.6; 21.1; 31.7 and $42.2 \mathrm{ng} / \mathrm{ml}$ ); SIR (blank; 12.3; 24.6; 36.9 and $49.2 \mathrm{ng} / \mathrm{ml}$ ); EVE (blank; 10.2; 20.4; 30.6 and $40.8 \mathrm{ng} / \mathrm{ml}$ ); MPA (blank; 1.88; 3.75; 5.63 and 7.50 $\mu \mathrm{g} / \mathrm{ml})$.

All specimens were aliquoted and stored at $-20^{\circ} \mathrm{C}$. To match the higher $\mathrm{C} 2$-values (blood concentration two hours after application), CSA dilutions were carried out with spiked whole blood up to $2000 \mathrm{ng} / \mathrm{ml}$, because the highest calibrator level reaches just $896 \mathrm{ng} / \mathrm{ml}$.

Precision tests were performed with drug-free whole blood samples from patients attending different clinical departments (internal medicine, surgery and gynecology). Enrichment with methanolic stock solutions of each drug yielded three ascending concentrations (lowest concentration $=$ LOQ concentration). Due to the large volume required ( $3 \mathrm{ml}$ for each drug and concentration add up to approx. $50 \mathrm{ml}$ in total), the assays were not performed with certified controls. Furthermore, the use of the patient material came closest to the clinical environment. After gentle mixing for four hours on a roller mixer, samples were aliquoted and stored at $-20^{\circ} \mathrm{C}$ until use.

For the intra-assay precision test, each concentration was extracted five times and measured in series.

The same specimens were used to detect the interassay precision on twenty continuous days (measured once), according to a new daily calibration.

To detect interference due to coeluted matrix constituents, the postcolumn infusion method as published by Taylor and Vogeser was implemented $[7,20]$. For this purpose methanolic solutions containing the immunosuppressants $(500 \mathrm{ng} / \mathrm{ml})$ were infused, while injecting analyte-free extracts of whole blood samples. Methanolic drug dilution was supplied at a constant flow rate of 10 $\mu \mathrm{l} / \mathrm{min}$ to the HPLC flow of $0.9 \mathrm{ml} / \mathrm{min}$ with a T-piece directly before the ESI source, resulting in a further dilution of 1:90. After injecting drug-free blood matrix or methanol, total ion count (TIC) of the particular drug was monitored during the entire analytical cycle.

Process efficiency and recovery were tested by means of addition at pre- and post-extraction steps. For each analyte before sample preparation, drug free blood was spiked with three concentrations of methanolic stock solutions (CSA: 10, 100, $500 \mathrm{ng} / \mathrm{ml}$, TAC, SIR, EVE: 2, 
5, $10 \mathrm{ng} / \mathrm{ml}$, and MPA: 0.5, 1, $5 \mu \mathrm{g} / \mathrm{ml})$. The results were related to extracts that were enriched with corresponding methanolic stock solutions.

The stability of the extracted samples was checked by repeated determination after five hours (four patient samples were used for each analyte). The samples were kept cool throughout this time period in the autosampler.

An integral element of further quality assurance was the continuous participation in the international proficiency-testing scheme (Prof. Holt, London). For CSA, TAC and SIR three samples were supplied each month, for EVE three samples six times per year and for MPA two samples four times per year. Each scheme included samples which were either spiked to a known concentration or were pooled from patients receiving the target drug. Aliquots of these samples were extracted and measured on a daily basis for five consecutive days.

\section{Results}

At the beginning of this validation, erythrocyte lysis was confirmed microscopically. In later tests it was checked only on a random basis. Ultrasonic treatment was introduced as a result of an inacceptable CV (> 15\%) for inter-assay precision at the beginning of the validation process. Without ultrasonic treatment lysis was incomplete, so that numerous red blood cells remained undamaged. However, the ultrasound treated samples were lysed completely (Figure 2).

The advantage of stable-isotope-labeled isoforms for use as IS was shown by the variant ionization behavior of analytes and the corresponding deuterated standards compared to CSD and ascomycin in two different lots of certified control materials. In this case the signal for CSA and CSA-d4 increased to a similar extent (by $100 \%)$ compared to the previous lot, resulting in a correct calculation of the target value. In contrast, the signal for CSD rose only by half, leading to a false ratio and overestimation of the CSA target value.

The patients' samples were also measured in parallel using CSD and CSA-d4 as IS. In some cases the values calculated with CSD differed by $60 \%-150 \%$ from the results obtained with CSA-d4. Comparable results could be obtained for ascomycin and TAC- ${ }^{13} \mathrm{C}-\mathrm{d}_{2}$, EVE- $\mathrm{d}_{4}$ and SIR- ${ }^{13} \mathrm{C}-\mathrm{d}_{3}$ respectively. Due to the experience with the certified control material and its known target values, further use of CSD and ascomycin was stopped.

A summary of validation results is depicted in Table 2 . All drugs revealed linear behavior up to the highest concentration of calibrator. Assay ranges were $2-1250 \mathrm{ng} /$ $\mathrm{ml}$ for CSA; $0.5-42.2 \mathrm{ng} / \mathrm{ml}$ for TAC; $0.6-49.2 \mathrm{ng} / \mathrm{ml}$ for SIR; $0.5-40.8 \mathrm{ng} / \mathrm{ml}$ for EVE and $0.01-7.5 \mu \mathrm{g} / \mathrm{ml}$ for MPA. The coefficient of determination $\left(\mathrm{r}^{2}\right)$ was at a minimum of 0.997. Assay sensitivity as well as data for precision and accuracy exceeded clinical requirements, as defined as a minimum sensitivity of at least $1 \mathrm{ng} / \mathrm{ml}$ for TAC, SIR and EVE, $10 \mathrm{ng} / \mathrm{ml}$ for CSA and $0.02 \mu \mathrm{g} /$ $\mathrm{ml}$ for MPA, and a CV for precision and accuracy below $20 \%$. Surprisingly, the CV for LOQ concentrations was lower than for intra-assay precision.

The calibration results are summarized in Table 3.

Recovery after sample preparation resulted in a process efficiency of $84 \pm 0.6 \%$ for TAC, $80.7 \pm 1.4 \%$ for EVE, $80.2 \pm 1.3 \%$ for CSA, $77.1 \pm 2.6 \%$ for SIR and 76.6 $\pm 1.9 \%$ for MPA.

Figure 3 shows exemple chromatograms of patient samples for each analyte and their corresponding IS. Additionally, common IS are compared with deuterated IS. All peaks exhibit a nearly symmetrical shape; retention of analytes and deuterated standards are concordant in time, whereas the common standards show a slight difference in retention time.

The additional rinsing of the SPE column with methanol for one minute, during separation in the analytical column, doubled the column lifetime to at least three months or 4500 analyses. The analytical quality, reflected by the shape of the peaks, remained stable throughout this time.

Figure 4 depicts the monitoring of matrix effects by means of post column infusion in conjunction with the corresponding retention times. The graphical time course of the total ion count is illustrated by the CSA figure. After the valve switch to methanolic buffer (A), the sample was flushed to the analytical column. Strong

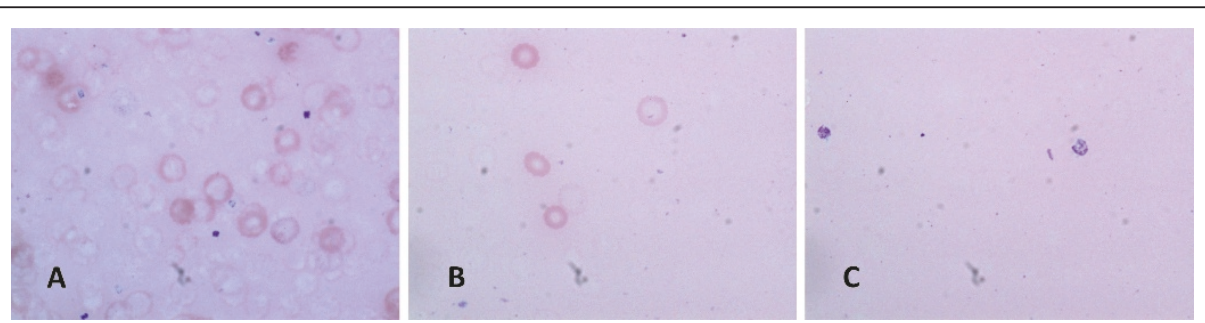

Figure 2 Microscopic images of whole blood $(50 \mu \mathrm{l})$ incubated with water $(125 \mu \mathrm{l})$ for various times. A: immediately after adding water B: 2 min incubation with water C: 2 min incubation with water plus $20 \mathrm{sec}$ of ultrasonic treatment. 
Table 2 Validation results

\begin{tabular}{|c|c|c|c|c|c|c|}
\hline Analyte & $\begin{array}{c}\text { Measuring Range (ng/ml) } \\
\text { MPA: } \mu \mathrm{g} / \mathrm{ml} \\
\mathrm{n}=3\end{array}$ & $\begin{array}{c}\text { LOQ, CV (\%) } \\
\text { Accuracy } \\
(\%) \\
\mathrm{n}=5 \\
\end{array}$ & $\begin{array}{l}\text { Sample Conc. } \\
\text { (ng/ml) } \\
\text { MPA: } \mu \mathrm{g} / \mathrm{ml}\end{array}$ & $\begin{array}{c}\text { Intra Assay Precision } \\
(\%) \\
n=5\end{array}$ & $\begin{array}{l}\text { Inter Assay Precision (\%) } \\
\qquad \mathrm{n}=20\end{array}$ & $\begin{array}{c}\text { Accuracy (\%) } \\
\text { Intra/Inter } \\
\text { Assay }\end{array}$ \\
\hline \multirow[t]{3}{*}{ Cyclosporine A } & $2-1250$ & 3.7 & 2 & 7.4 & 7.1 & $125 / 113$ \\
\hline & $r^{2}=0.997$ & 104 & 100 & 2.3 & 2.9 & 104/106 \\
\hline & & & 500 & 0.9 & 2.5 & $106 / 105$ \\
\hline \multirow[t]{3}{*}{ Tacrolimus } & $0.5-42.2$ & 7.5 & 0.5 & 12.3 & 9.1 & 138/100 \\
\hline & $r^{2}=0.998$ & 108 & 10 & 3.7 & 4.9 & 116/99 \\
\hline & & & 20 & 3.8 & 4.5 & $111 / 98$ \\
\hline \multirow[t]{3}{*}{ Sirolimus } & $0.62-49.2$ & 4.6 & 1 & 12.0 & 11.5 & 125/107 \\
\hline & $r^{2}=0.999$ & 106 & 10 & 5.1 & 5.0 & $100 / 102$ \\
\hline & & & 20 & 5.0 & 4.9 & 108/102 \\
\hline \multirow[t]{3}{*}{ Everolimus } & $0.53-40.8$ & 6.4 & 1 & 14.7 & 12.5 & $89 / 90$ \\
\hline & $r^{2}=0.999$ & 118 & 10 & 2.9 & 6.9 & $97 / 96$ \\
\hline & & & 20 & 5.1 & 5.7 & $102 / 97$ \\
\hline \multirow[t]{3}{*}{ MPA } & $0.01-7.5$ & 7.0 & 0.02 & 11.0 & 9.8 & $97 / 102$ \\
\hline & $r^{2}=0.999$ & 105 & 0.2 & 1.3 & 3.2 & 97/97 \\
\hline & & & 5 & 1.6 & 3.0 & $102 / 99$ \\
\hline
\end{tabular}

(LOQ: low limit of quantification, CV: variation coefficient)

ion loss occurred during the following elution of aqueous buffer from the SPE column to the analytical column (C). Reconditioning of the SPE column (B) started at $1.9 \mathrm{~min}$, when the valve switched back to position $\mathrm{A}$.

A second signal loss appeared during the assumed elution of sample matrix (D).

Compared to methanol-based solutions, the three tested dilutions of sample preparation exhibit distinct matrix effects (Figure 4). As expected, these effects are more pronounced at a lower sample dilution (1:3). Surprisingly, the higher dilution of 1:21 resulted in stronger matrix effects than the lower dilution of $1: 11$. Due to a lack of significant reduction of matrix effects and increased imprecision at higher dilutions, the 1:11 ratio was chosen for further development of this application.

The time frame of ion suppression ranged from 2.15 to $2.5 \mathrm{~min}$. With $2.21 \mathrm{~min}$., the retention time of CSA was within this time window, whereas TAC, SIR and EVE were not affected (retention time: $2.10 \mathrm{~min}$.). As illustrated in terms of quality, CSA showed a high and TAC a slight ion suppression, whereas SIR, EVE and in MPA exhibited an ion enhancement. These effects were offset by the deuterated internal standards.

Figure 5 depicts the distinct chromatographic separation of MPAG and MPA. The in-source fragmentation of MPAG to MPA is shown by the signal of the $\mathrm{m} / \mathrm{z}$ 338.1/207.1 trace within the MPAG peak. Concerning MPA, comparative measurements of mass spectrometry and immunoassay showed significantly different results for samples with a high content of MPAG (data not shown). This discrepancy of up to $50 \%$ reflects, among other things, a high cross-reactivity of the antibody against the glucuronide.

Sample stability, as confirmed by repeated measurements of 15 extracted patient samples per analyte after 5 hours, resulted in a $\mathrm{CV}<8 \%$.

Participation in the international proficiency testing scheme in 2010 (analytical services international ASI, Prof. Holt, U.K) revealed a mean accuracy of 3.6\% for CSA, $10.2 \%$ for TAC, $-3.5 \%$ for SIR, $2.1 \%$ for EVE and $-2.6 \%$ for MPA and confirmed the validity of the method.

\section{Discussion}

When determining immunosuppressants in whole blood, thorough sample preparation plays a crucial role. Complete lysis of erythrocytes and protein precipitation are mandatory for good reproducibility. We employed water incubation in conjunction with ultrasound treatment to ensure complete lysis, which was microscopically verified at the beginning of the validation process and ensured high reproducibility of the measurements. Water lysis alone left many erythrocytes undamaged and compromised precision.

Second, internal standards play a key role in massspectrometric analyses. The use of CSD, ascomycin and MPAC as internal standards for the quantification of immunosuppressants can cause measurement errors due to an ionization process in the ESI source which deviates from that of the analyte [6,7,20-22]. Initially, the common standards were also used for this validation protocol. However, especially for CSA, divergence of 
Table 3 Calibration results (Intra Assay Precision)

\begin{tabular}{|c|c|c|c|c|}
\hline Analyte & $\begin{array}{c}\text { Target Value } \\
\text { (ng/ml) } \\
\text { MPA } \\
(\mu \mathrm{g} / \mathrm{ml})\end{array}$ & $\begin{array}{c}\begin{array}{c}\text { Measured Value } \\
(\mathrm{ng} / \mathrm{ml}) \\
\mathrm{MPA} \\
(\mu \mathrm{g} / \mathrm{ml})\end{array} \\
\end{array}$ & Accuracy (\%) & $\begin{array}{l}\text { Linear Regression } \\
\text { (1/x weighting) }\end{array}$ \\
\hline \multirow[t]{7}{*}{ Cyclosporine A } & 0 & $<0$ & N/A & $y=0.00419 x+0.000559$ \\
\hline & 23.5 & 24.5 & 104 & $r=0.9996$ \\
\hline & 127 & 121 & 94.9 & \\
\hline & 299 & 291 & 97.3 & \\
\hline & 484 & 506 & 105 & \\
\hline & 703 & 714 & 102 & \\
\hline & 896 & 877 & 97.8 & \\
\hline \multirow[t]{7}{*}{ Tacrolimus } & 0 & $<0$ & N/A & $y=0.145 x+0.00678$ \\
\hline & 2.1 & 2.45 & 117 & $r=0.9994$ \\
\hline & 5.8 & 6.06 & 105 & \\
\hline & 12.1 & 12.3 & 102 & \\
\hline & 18.1 & 18.7 & 103 & \\
\hline & 24.4 & 24.2 & 99.3 & \\
\hline & 42.4 & 41.1 & 97.0 & \\
\hline \multirow[t]{7}{*}{ Sirolimus } & 0 & $<0$ & N/A & $y=0.104 x+0.0171$ \\
\hline & 2.6 & 2.25 & 86.7 & $r=0.9996$ \\
\hline & 6.6 & 6.21 & 94.2 & \\
\hline & 12.8 & 13.0 & 102 & \\
\hline & 20.0 & 20.8 & 104 & \\
\hline & 29.0 & 28.9 & 99.5 & \\
\hline & 49.2 & 49.1 & 99.7 & \\
\hline \multirow[t]{7}{*}{ Everolimus } & 0 & $<0$ & $\mathrm{~N} / \mathrm{A}$ & $y=0.112 x+9.76 e-0.09$ \\
\hline & 2.2 & 2.25 & 102 & $r=0.9992$ \\
\hline & 5.9 & 5.66 & 95.8 & \\
\hline & 11.6 & 11.2 & 96.3 & \\
\hline & 17.0 & 18.0 & 106 & \\
\hline & 23.2 & 24.0 & 103 & \\
\hline & 40.8 & 39.6 & 97 & \\
\hline MPA & 4.27 & \multicolumn{2}{|c|}{$\begin{array}{l}\text { 1-point calibration } \\
\text { linear through zero }\end{array}$} & $\begin{array}{c}y=0.00494 x \\
r=1.0\end{array}$ \\
\hline
\end{tabular}

ionization efficiency (analyte vs. IS) were so large that the results were significantly distorted.

This effect became particularly evident in matrices of two certified control materials. In this case, CSA was subject to ion enhancement, resulting in $40 \%$ higher values than the specified target. The use of deuterated standards could compensate for these matrix effects and resulted in correct recovery. One batch of calibrators was also affected by this phenomenon. In routine clinical measurements, matrix impacts based on (non-standardized) patient samples will always be a recurrent problem, which could be overcome by the use of deuterated standards.

Crucial for correct recovery is an IS which comes chemically as close as possible to the analyte. The close chemical relationship leads to identical retention time, so that IS and analyte are affected in the same way, and matrix effects can be compensated for.
Ascomycin also exhibited an elution behavior dependent on the sample matrix, making it liable to wide fluctuations. By use of deuterated standards that eluted concurrently with the analytes, we noted that measurement errors, especially those caused by ion suppression or enhancement, were nearly all compensated for.

In contrast to other laboratories, the working solution for sample preparation was set up daily to avoid any instability. Small sample batches contributed to a fast processing time, which could be kept below one hour. Repeated analyses of samples after five hours confirmed the previous results and thus verified the stability of the extracted samples.

The analysis time required for this method is $3.5 \mathrm{~min}$ utes. We are highly suspicious of attempts to shorten analysis times to one minute [8], especially when dealing with substances having a variety of metabolites like CSA, as isobars may appear which interfere with the 

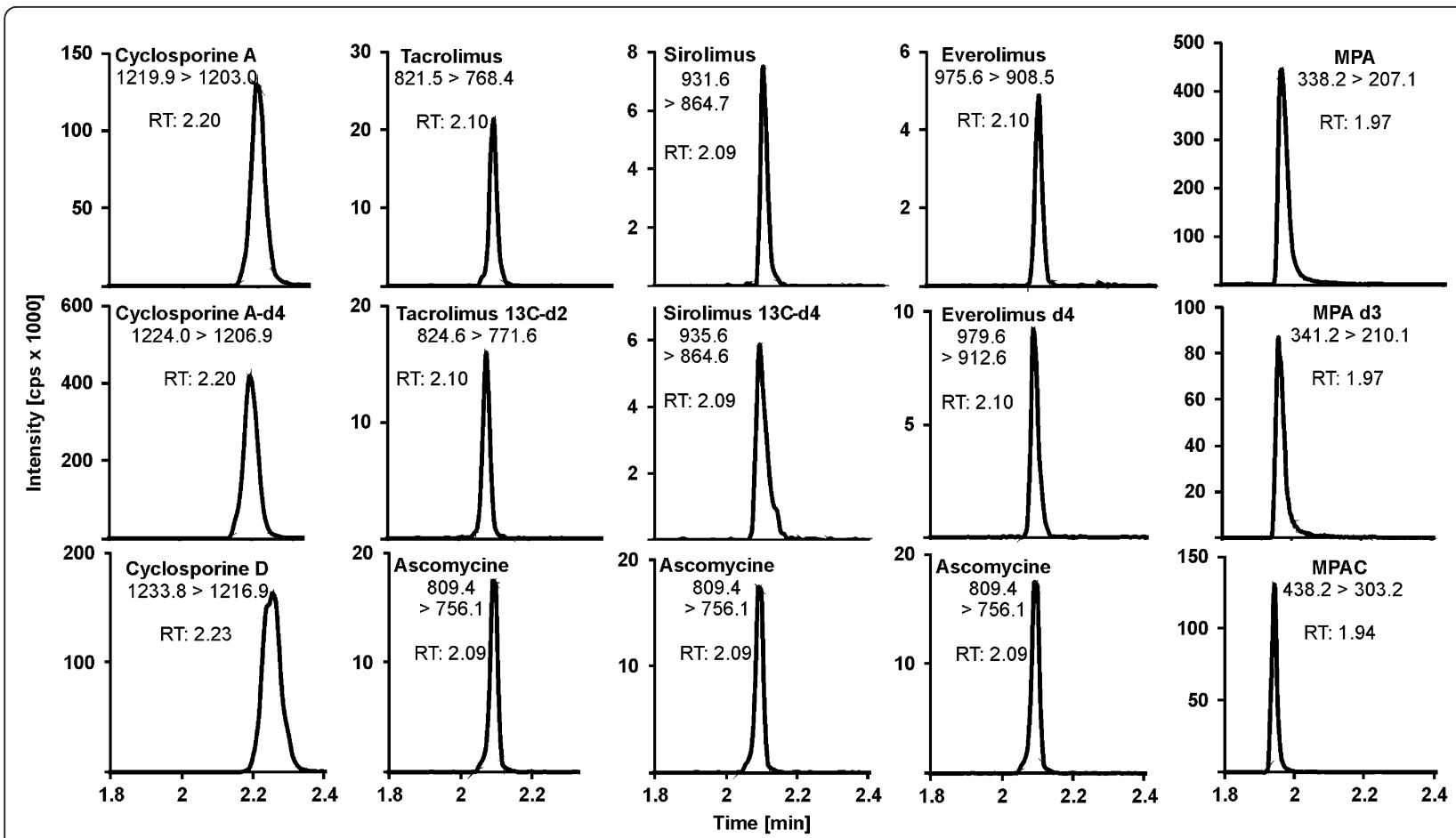

Figure 3 Exemplary chromatograms of original routine patient samples containing $103 \mathrm{ng} / \mathrm{ml} \mathrm{CSA,} 9.6 \mathrm{ng} / \mathrm{ml}$ TAC, $6.1 \mathrm{ng} / \mathrm{ml} \mathrm{SIR,} 5.3$ $\mathrm{ng} / \mathrm{ml}$ EVE and $4.9 \mu \mathrm{g} / \mathrm{ml}$ MPA. Mass transitions are stated.

analyte, skewing the results. The likelihood of such interferences can be minimized by thorough HPLC separation.

One issue in developing this application was the identification of the most appropriate sample dilution. The initial dilution of 1:3 was raised to $1: 21$ so as to reduce matrix effects and soiling of the equipment [17]. Later, the dilution was reduced to $1: 11$ due to the observation that higher dilutions failed in further reduction of matrix effects and lead rather to greater imprecision, in spite of the equipment's sensitivity.

Precision was not only influenced by dilution but also depended on the material used. Certified lyophilized control material used for the determination of LOQ revealed a lower $\mathrm{CV}$ than spiked patient's blood for intra- and inter-assay testing. This phenomenon may possibly be explained by the different matrices.

Another factor of influence was the purity of the reagents. Many annoying adducts became apparent in water and methanol when using HPLC-grade reagents. These adducts have considerably reduced the sensitivity.

As was noted by Annesley [23], we also observed significant differences in signal strengths from the same sample, even when using several LC/MS grades of methanol from various companies.

Most laboratories employ an HPLC-UV method or immunoassay to determine MPA. Our method herein stands out not just because CSA, TAC, SIR and EVE can all be measured in parallel, but because MPA can also be quantified using the same application. However, due to the fact that no commercial calibrator is available containing MPA, it is inevitable that a separate calibration is necessary. The additional expenditure is compensated by identical analytical conditions and procedures for all analytes. In agreement with results from other working groups, we failed to determine MPA in whole blood due to inadequate precision and accuracy (personal communication). Currently there is no explanation for this result. As a consequence, sample preparation must be carried out with plasma. However, additional UV-HPLC analysis for the determination of MPA, as is customary in most laboratories, can be waived.

As mentioned above, the chromatographic separation of MPAG and MPA is crucial due to in-source fragmentation of MPAG to MPA, which is then added to the intrinsic value of MPA in the case of coelution [11]. The extent to which this secession occurs is essentially construct-dependent. Glucuronide splits off to such a miniscule degree on our equipment that we could forgo chromatographic separation, although for reasons of safety, we do not. Different retention times for MPA and MPAG could be realized by a flow-through procedure on the online-SPE column. 


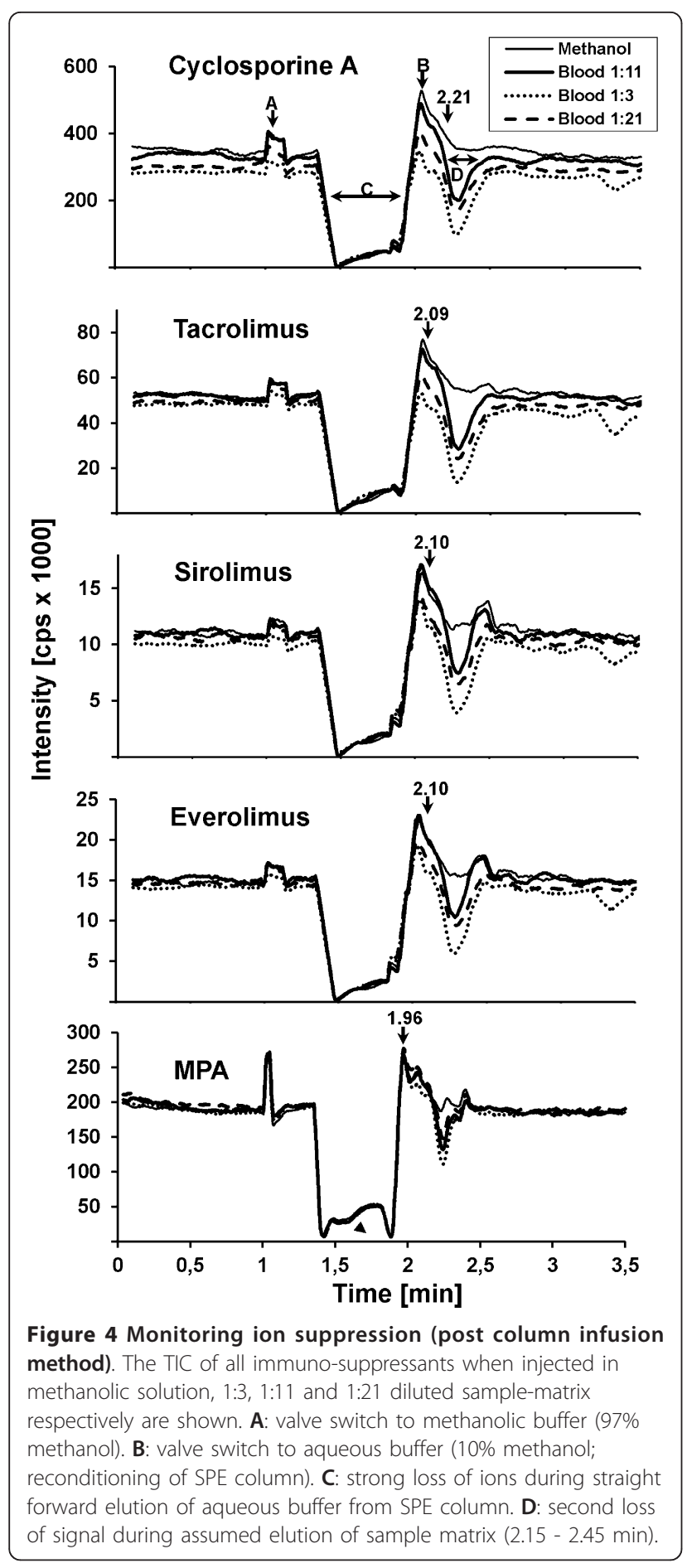

Adequate separation of MPA from MPAG proved impossible when we tried to shorten the analysis time to 2.5 minutes or using the back flush online SPE method.

We compared our method to LC-MS/MS methods used by two university and commercial laboratories, observing that our results differed strikingly from the latter. This clearly illustrates that it is not just the

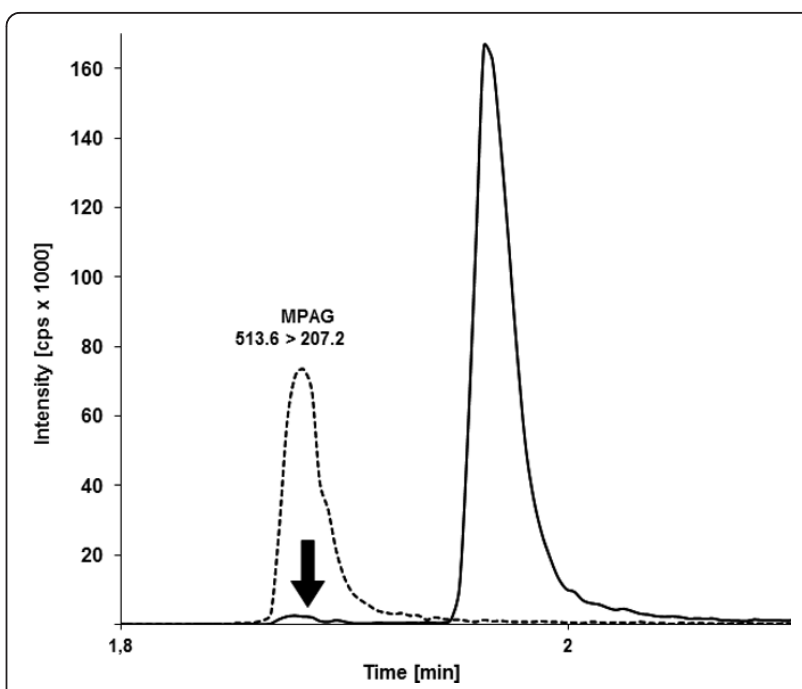

Figure 5 Chromatographic separation of MPAG m/z 513.6/ 207.2 (dotted line) and MPA $\mathrm{m} / \mathrm{z} 338.2 / 207.1$ (solid line). The arrow indicates the in-source fragmentation of MPAG to MPA.

immunoassays which need to be standardized - the physical methods must be standardized as well in order to guarantee comparability.

The wide divergence of LC-MS methods is reflected in the international proficiency testing scheme's evaluation and is caused by differences in equipment, applications, and sample preparation. Furthermore, this application is regularly confirmed via national proficiency testing (DGKL, Instand).

Surprisingly, the signal strengths of the analytes and the IS differ significantly even when identically-constructed instruments from the same manufacturer are used, because of the sources' divergent ionization behavior. By changing the heating units and probes we observed an up to a ten-fold difference in signal strengths when comparing three sources on two mass spectrometers. At this point, the manufacturer has a duty to develop better standardization procedures in order to improve quality control. Devices should not be tested with a reference source, but with the supplied sources.

The prevalence of immunosuppressive combination therapy allows dose reduction and minimizes undesirable side effects $[2,24]$. However, it makes greater demands on the analysis systems used, especially concerning precision and reproducibility [19]. This explains why replacement of immunoassays by mass spectrometry is taking root - it offers significantly better sensitivity and is highly specific for the drug's parent compound, without having cross reactivity to metabolites. In the case of CSA, the average recovery of immunoassays is up to $25 \%$ higher compared to mass spectrometry. This difference is due to individually occurring metabolites with unknown pharmacologic effects. 
In addition, economic considerations also favor the mass-spectrometry based method. First, it is time saving since a single analytical run is adequate for the determination of CSA, TAC, SIR and/or EVE in patients with combination therapy. Although two analytical runs are needed in the case of MPA co-administration, the saving in time is still evident. Second, the authors were able to reduce the reagent and consumable costs by more than $80 \%$ compared to the previously used immunoassays (340,000 Euro/year for CSA, TAC and MPA vs. 50,000 Euro/year for all analytes).

Arguments against mass spectrometry are its high acquisition costs, high skill level required of personnel, and the complex validation process necessary, so that academic expertise is indispensable. Moreover, in the case of defects or maintenance, the laboratory may require a back-up system.

But nevertheless, once the mass-spectrometric method has been established, it is easy to use in daily routine. Therefore, the authors recommend this method for laboratories with high throughput such as university hospitals with transplantation departments.

\section{Conclusion}

Before mass spectrometric applications can be employed for routine clinical purposes or be considered to be a gold standard, they must be subjected to a thorough validation process. The use of deuterated internal standards is highly recommended since the commonly used standards may cause analytical problems due to an ionization efficiency differing from that of the analyte.

Last but not least: without established standards the findings from different laboratories cannot be reliably compared.

\section{Acknowledgements}

We would like to thank Dragan Zloporubovic for excellent technical support and Gerhard Pütz and Marie Follo for valuable discussions and proofreading of the manuscript.

\section{Authors' contributions}

$A B$ and TE were responsible for the development of sample preparation, chromatography conditions and mass-spectrometer settings. KW participated in the statistical analysis of the results. All authors contributed to the content and review of the manuscript and read and approved the final manuscript.

\section{Competing interests}

The authors declare that they have no competing interests.

Received: 14 October 2011 Accepted: 11 January 2012

Published: 11 January 2012

\section{References}

1. Beckebaum S, Armstrong VW, Cicinnati VR, Streit F, Klein CG, Gerken G, Paul A, Oellerich M: Pharmacokinetics of mycophenolic acid and its glucuronide metabolites in stable adult liver transplant recipients with renal dysfunction on a low-dose calcineurin inhibitor regimen and mycophenolate mofetil. Ther Drug Monit 2009, 31(2):205-210.
2. Ekberg H, Tedesco-Silva H, Demirbas A, Vitko S, Nashan B, Gurkan A Margreiter R, Hugo C, Grinyo JM, Frei U, et al: Reduced exposure to calcineurin inhibitors in renal transplantation. N Engl J Med 2007, 357(25):2562-2575

3. Srinivas TR, Meier-Kriesche HU: Minimizing immunosuppression, an alternative approach to reducing side effects: objectives and interim result. Clin J Am Soc Nephrol 2008, 3(Suppl 2):S101-116.

4. Ghoshal AK, Soldin SJ: IMx tacrolimus II assay: is it reliable at low blood concentrations? A comparison with tandem MS/MS. Clin Biochem 2002, 35(5):389-392.

5. Schneider HSW: Tandem-Massenspektrometrie in der therapeutischen Arzneimittelspiegel-Bestimmung: Praxis und Tücken der Anwendung. J Lab Med 2006, 6(30):428-437.

6. Seger CVM: Immunosuppressant drug monitoring - a routine undertaking? J Lab Med 2010, 3(34):117-128.

7. Taylor PJ: Matrix effects: the Achilles heel of quantitative highperformance liquid chromatography-electrospray-tandem mass spectrometry. Clin Biochem 2005, 38(4):328-334.

8. Meinitzer A, Gartner G, Pilz S, Stettin M: Ultra fast liquid chromatographytandem mass spectrometry routine method for simultaneous determination of cyclosporin A, tacrolimus, sirolimus, and everolimus in whole blood using deuterated internal standards for cyclosporin A and everolimus. Ther Drug Monit 2010, 32(1):61-66.

9. O'Halloran S, llett KF: Evaluation of a deuterium-labeled internal standard for the measurement of sirolimus by high-throughput HPLC electrospray ionization tandem mass spectrometry. Clin Chem 2008, 54(8):1386-1389.

10. Taylor PJ, Brown SR, Cooper DP, Salm P, Morris MR, Pillans PI, Lynch SV: Evaluation of 3 internal standards for the measurement of cyclosporin by HPLC-mass spectrometry. Clin Chem 2005, 51(10):1890-1893.

11. Annesley TM, Clayton LT: Quantification of mycophenolic acid and glucuronide metabolite in human serum by HPLC-tandem mass spectrometry. Clin Chem 2005, 51(5):872-877.

12. Ceglarek U, Casetta B, Lembcke J, Baumann S, Fiedler GM, Thiery J: Inclusion of MPA and in a rapid multi-drug LC-tandem mass spectrometric method for simultaneous determination of immunosuppressants. Clin Chim Acta 2006, 373(1-2):168-171.

13. Bogusz MJ, Enazi EA, Hassan H, Abdel-Jawaad J, Ruwaily JA, Tufail MA: Simultaneous LC-MS-MS determination of cyclosporine A, tacrolimus, and sirolimus in whole blood as well as mycophenolic acid in plasma using common pretreatment procedure. J Chromatogr B Analyt Technol Biomed Life Sci 2007, 850(1-2):471-480.

14. Ceglarek U, Lembcke J, Fiedler GM, Werner M, Witzigmann H, Hauss JP, Thiery J: Rapid simultaneous quantification of immunosuppressants in transplant patients by turbulent flow chromatography combined with tandem mass spectrometry. Clin Chim Acta 2004, 346(2):181-190.

15. Seger C, Tentschert K, Stoggl W, Griesmacher A, Ramsay SL: A rapid HPLCMS/MS method for the simultaneous quantification of cyclosporine $A$, tacrolimus, sirolimus and everolimus in human blood samples. Nat Protoc 2009, 4(4):526-534

16. Koster RA, Dijkers EC, Uges DR: Robust, high-throughput LC-MS/MS method for therapeutic drug monitoring of cyclosporine, tacrolimus, everolimus, and sirolimus in whole blood. Ther Drug Monit 2009, 31(1):116-125.

17. Annesley TM, Clayton L: Simple extraction protocol for analysis of immunosuppressant drugs in whole blood. Clin Chem 2004, 50(10):1845-1848.

18. US Department of Health and Human Services FaDA, Reserach CfDEa: Bioanalytical method validation. 2001.

19. Wayne P: Evaluation of precision performance of clinical chemistry devices. National Committee for Clinical Laboratory Standards 1992.

20. Vogeser M, Seger C: Pitfalls associated with the use of liquid chromatography-tandem mass spectrometry in the clinical laboratory. Clin Chem 2010, 56(8):1234-1244.

21. Chambers E, Wagrowski-Diehl DM, Lu Z, Mazzeo JR: Systematic and comprehensive strategy for reducing matrix effects in LC/MS/MS analyses. J Chromatogr B Analyt Technol Biomed Life Sci 2007, 852(12):22-34.

22. Vogeser M: Instrument-specific matrix effects of calibration materials in the LC-MS/MS analysis of tacrolimus. Clin Chem 2008, 54(8):1406-1408.

23. Annesley TM: Methanol-associated matrix effects in electrospray ionization tandem mass spectrometry. Clin Chem 2007, 53(10):1827-1834 
24. Wallemacq $P$, Armstrong WW, Brunet $M$, Haufroid V, Holt DW, Johnston A, Kuypers D, Le Meur Y, Marquet P, Oellerich M, et al: Opportunities to optimize tacrolimus therapy in solid organ transplantation: report of the European consensus conference. Ther Drug Monit 2009, 31(2):139-152.

\section{Pre-publication history}

The pre-publication history for this paper can be accessed here: http://www.biomedcentral.com/1472-6904/12/2/prepub

doi:10.1186/1472-6904-12-2

Cite this article as: Buchwald et al:: Validation of an LC-MS/MS method

to determine five immunosuppressants with deuterated internal

standards including MPA. BMC Clinical Pharmacology 2012 12:2.

Submit your next manuscript to BioMed Central and take full advantage of:

- Convenient online submission

- Thorough peer review

- No space constraints or color figure charges

- Immediate publication on acceptance

- Inclusion in PubMed, CAS, Scopus and Google Scholar

- Research which is freely available for redistribution

Submit your manuscript at www.biomedcentral.com/submit 